\title{
A HAUSDORFF TOPOLOGY FOR THE CLOSED SUBSETS OF A LOCALLY COMPACT NON-HAUSDORFF SPACE
}

\author{
J. M. G. FELL
}

In the structure theory of $C^{*}$-algebras an important role is played by certain topological spaces $X$ which, though locally compact in a certain sense, do not in general satisfy even the weakest separation axiom. This note is concerned with the construction of a compact Hausdorff topology for the space $\mathfrak{e}(X)$ of all closed subsets of such a space $X$. This construction occurs naturally in the theory of $C^{*}$. algebras; but, in view of its purely topological nature, it seemed wise to publish it apart from the algebraic context. ${ }^{1}$

A comparison of our topology with the topology of closed subsets studied by Michael in [2] will be made later in this note.

For the theory of nets we refer the reader to [1]. A net $\left\{x_{\nu}\right\}$ is universal if, for every set $A, x_{\nu}$ is either $\nu$-eventually in $A$ or $\nu$-eventually outside $A$. Every net has a universal subnet. By the limit set of a net $\left\{x_{\nu}\right\}$ of elements of a topological space $X$ we mean the set of those $y$ in $X$ such that $\left\{x_{\nu}\right\}$ converges to $y$; the net $\left\{x_{\nu}\right\}$ is primitive if the limit set of $\left\{x_{\nu}\right\}$ is the same as the limit set of each subnet of $\left\{x_{\nu}\right\}$, i.e., if every cluster point of the net is also a limit of the net. A universal net is obviously primitive. In a locally compact Hausdorff space $X$ the primitive nets are just those which converge either to some point of $X$ or to the point at infinity.

An arbitrary topological space $X$ will be called locally compact if, to every point $x$ of $X$ and every neighborhood $U$ of $x$, there is a compact neighborhood of $x$ contained in $U$. A compact Hausdorff space is of course locally compact; but a compact non-Hausdorff space need not be locally compact.

Let $X$ be any fixed topological space (no separation axioms being assumed), and let $\mathrm{e}(X)$ be the family of all closed subsets of $X$ (including the void set $\Lambda$ ). For each compact subset $C$ of $X$, and each finite family $\mathfrak{F}$ of nonvoid open subsets of $X$, let $U(C ; \mathfrak{F})$ be the subset of $\mathfrak{e}(X)$ consisting of all $Y$ such that (i) $Y \cap C=\Lambda$, and (ii) $Y \cap A \neq \Lambda$ for each $A$ in $\mathcal{F}$. A subset $W$ of $\mathfrak{e}(X)$ is open if it is a union of certain of the $U(C ; \Im)$. It is easily verified that this notion of openness defines a topology for $\mathfrak{e}(X)$, which we will call the $H$-topology.

Received by the editors April 4, 1961.

${ }^{1}$ The application to $C^{*}$-algebras is given in our article The structure of algebras of operator fields, Acta Math. 106 (1961), 233-280. The author wishes to thank Professor $\mathrm{H}$. Corson for a discussion which clarified the ideas of this note. This research was supported in part by Research Grant NSF G11322. 
Lemma 1. $\mathrm{e}(X)$ is compact in the H-topology.

Proof. Let $\left\{Y^{i}\right\}$ be a universal net of elements of $\mathfrak{e}(X)$, and define $Z$ to be the set of those $x$ in $X$ such that, for each neighborhood $U$ of $x, Y^{i} \cap U \neq \Lambda$ for all large enough $i$. Obviously $Z \in \mathcal{C}(X)$. It will be sufficient to show that $Y^{i} \rightarrow Z$ in the $H$-topology.

Let $U(C ; F)$ be a typical neighborhood of $Z(C$ and $F$ being as before). For each $A$ in $\mathcal{F}$ there is an element $x$ of $Z \cap A$; and the definition of $Z$ gives

$$
Y^{i} \cap A \neq \Lambda \text { for all large enough } i .
$$

Now suppose it were false that

$$
Y^{i} \cap C=\Lambda \text { for all large enough } i .
$$

Then by the universality of $\left\{Y^{i}\right\}, Y^{i} \cap C \neq \Lambda$ for all large enough $i$. Choosing an element $x_{i}$ of $Y_{i} \cap \mathcal{C}$ for each large enough $i$, we have by the compactness of $C$ (passing to a subnet if necessary) $x_{i} \rightarrow z$ for some $z$ in $C$. So, for each neighborhood $V$ of $z, Y^{i} \cap V \neq \Lambda$ for all large enough $i$; whence $z \in Z$, or $C \cap Z \neq \Lambda$. This is impossible since $Z \in U(C ; F)$; so (2) is proved. By (1) and (2) and the arbitrariness of $U(C ; \mathcal{F})$, we have $Y^{i} \rightarrow Z$.

Theorem 1. If $X$ is locally compact, $\mathfrak{e}(X)$ with the H-topology is a compact Hausdorff space.

Proof. Let $Y_{1}$ and $Y_{2}$ be distinct elements of $\mathfrak{e}(X)$, and suppose $x \in Y_{1}-Y_{2}$. By local compactness there is a compact neighborhood $V$ of $x$ for which $V \cap Y_{2}=\Lambda$; thus $Y_{2} \in U(V ; \Lambda)$. Clearly $Y_{1} \in U\left(\Lambda ;\left\{V^{\prime}\right\}\right)$, where $V^{\prime}$ is the interior of $V$. Since $U(V ; \Lambda)$ and $U\left(\Lambda ;\left\{V^{\prime}\right\}\right)$ are disjoint, $Y_{1}$ and $Y_{2}$ have disjoint neighborhoods. So $\mathfrak{C}(X)$ is Hausdorff. It is compact by Lemma 1 .

It may be well at this point to contrast our $H$-topology with the "finite topology" of Michael [2, p. 153]. The latter is defined by a basis consisting of sets $U(C ; \mathfrak{F})$ similar to ours except that the $C$ are required to be closed instead of compact. This makes a considerable difference in the properties of the two topologies. The properties of the "finite topology" parallel those of $X$ more closely than do those of the $H$-topology. For example, the "finite topology" is Hausdorff if and only if $X$ is regular [2, Theorem 4.9.3]. On the other hand, the $H$-topology is Hausdorff whenever $X$ is locally compact, no matter how badly unseparated $X$ may be; while if $X$ is not locally compact, it may even be metrizable (say an infinite-dimensional Banach space) without the $H$-topology being Hausdorff. Again, if $X$ is locally compact and $T_{1}$ but not Hausdorff, the map $x \rightarrow\{x\}$ will not be a homeo- 
morphism with respect to the $H$-topology, so that the latter is not "admissible" in the sense of [2, p. 153]. Needless to say, if $X$ is compact and Hausdorff, the "finite" and $H$-topologies coincide.

We shall henceforth always assume that $X$ is locally compact, and that $\mathfrak{C}(X)$ is equipped with the $H$-topology. Here are a few remarks on the $H$-topology, whose proof is left to the reader:

(I) The operation of union (carrying $A, B$ into $A \cup B$ ) is continuous on $\mathfrak{e}(X) \times \mathfrak{e}(X)$ into $\mathfrak{e}(X)$. Not so with intersection, however.

(II) If $Y \in \mathfrak{e}(X)$, the topology of $\mathfrak{e}(Y)$ relativized from $\mathfrak{e}(X)$ is the $H$-topology of $\mathfrak{e}(Y)$.

(III) Let $\alpha$ be an infinite cardinal. If $X$ has a basis for its open sets of cardinality no greater than $\alpha$, then $\mathfrak{e}(X)$ has a basis for its open sets of cardinality no greater than $\alpha$.

(IV) If $X$ is a locally compact topological group, the family $\mathcal{S}$ of all closed subgroups of $X$ is a closed subfamily of $\mathfrak{e}(X)$. Thus the $H$ topology relativized to $S$ is compact and Hausdorff.

We shall now consider an important subset of $\mathfrak{e}(X)$. For each $x$ in $X$, let $\langle x\rangle$ denote the closure in $X$ of the one-element set $\{x\}$. Let $\mathfrak{H C}(X)$ be the closure in $\mathfrak{e}(X)$ of the set of all $\langle x\rangle$, where $x$ ranges over $X$. By Theorem $1, \mathfrak{H C}(X)$ is a compact Hausdorff space, and the image of $X$ under the map $x \rightarrow\langle x\rangle$ is dense in $\operatorname{HC}(X)$.

We refer to $\mathscr{H C}(X)$ as the Hausdorff compactification of $X$. For obtaining its topology the following lemma is useful:

LEMMA 2. Let $\left\{x_{\nu}\right\}$ be a net of elements of $X$, and $Y$ a closed subset of $X$. The following two conditions are equivalent:

(i) $\left\{x_{\nu}\right\}$ is primitive, and $Y$ is its limit set;

(ii) $\lim _{\nu}\left\langle x_{\nu}\right\rangle=Y$ in $\mathrm{e}(X)$.

Proof. Assume (i); and let $U(C ; \mathfrak{F})$ be a neighborhood of $Y$. If $A \in \mathcal{F}$ and $x \in A \cap Y$, then $x_{\nu} \rightarrow x$, so

$$
\left\langle x_{\nu}\right\rangle \cap A \neq \Lambda \quad \nu \text {-eventually. }
$$

Suppose it is false that

$$
\left\langle x_{\nu}\right\rangle \cap C=\Lambda \quad \nu \text {-eventually. }
$$

Then there is a subnet $\left\{y_{\mu}\right\}$ of $\left\{x_{\nu}\right\}$ such that $\left\langle y_{\mu}\right\rangle \cap C \neq \Lambda$; let $z_{\mu} \in\left\langle y_{\mu}\right\rangle \cap C$. Passing again to a subnet, we may assume $z_{\mu} \rightarrow z, z \in C$. Now each open neighborhood of $z$ contains $z_{\mu}$, hence intersects $\left\langle y_{\mu}\right\rangle$, hence contains $y_{\mu}$, for all large enough $\mu$. Thus $y_{\mu} \rightarrow z$; so that by the primitivity of $\left\{x_{\nu}\right\}$ we have $z \in Y, Y \cap C \neq \Lambda$. This contradicts the fact that $Y \in U(C ; F)$; so (4) is proved. Now (3) and (4) and the arbitrariness of $U(C ; \mathcal{F})$ establish (ii).

Now assume (ii). Let $y_{\mu} \rightarrow y$, where $\left\{y_{\mu}\right\}$ is a subnet of $\left\{x_{\nu}\right\}$; and 
suppose $y \notin Y$. Then there is a compact neighborhood $W$ of $y$ such that $Y \in U(W ; \Lambda)$. By (ii) $y_{\mu} \in W$ for all large enough $\mu$, which is impossible since $y_{\mu} \rightarrow y$. Therefore $Y$ contains every cluster point of $\left\{x_{\nu}\right\}$. We shall complete the proof of (i) by showing that $Y$ is contained in the limit set of $\left\{x_{\nu}\right\}$. Let $y$ be in $Y$, and $A$ be any open neighborhood of $y$. Then $Y \in U(\Lambda ;\{A\})$; so that by (ii) $x_{\nu} \in A$ for all large enough $\nu$. Thus $x_{p} \rightarrow y$.

Corollary. The elements of $\mathfrak{H}(X)$ are precisely the limit sets of primitive nets of elements of $X$.

Here are a few easily verified examples of the Hausdorff compactification:

(I) If $X$ is compact and Hausdorff, $\mathfrak{F}(X)$ and $X$ are the same (if we identify $x$ and $\{x\})$. If $X$ is locally compact and Hausdorff but not compact, $\mathfrak{F}(X)$ is the one-point compactification of $X$ (the void set in $\mathfrak{e}(X)$ being the point at infinity).

(II) Let $X$ be the closed interval $[0,1]$ together with an extra "zero" 0 '. A subset $A$ of $X$ is to be open if (i) $A \cap[0,1]$ is open in the usual sense, and (ii) if $0^{\prime} \in A$, then $A$ contains the open interval $(0, \epsilon)$ for some $\epsilon>0$. This defines a locally compact non-Hausdorff topology for $X . \mathcal{H C}(X)$ consists of the one-element sets together with the two-element set $\left\{0,0^{\prime}\right\}$; it is homeomorphic with the space $S$ consisting of the ordinary Euclidean closed interval $[0,1]$ together with two isolated points $p$ and $q$. The homeomorphism is implemented by the map of $\mathscr{H C}(X)$ onto $S$ which sends $\{r\}$ into $r$ for $0<r \leqq 1$, $\left\{0,0^{\prime}\right\}$ into $0,\{0\}$ into $p$, and $\left\{0^{\prime}\right\}$ into $q$.

(III) Let $X$ be the square $\{(x, y) \mid 0 \leqq x \leqq 1,0 \leqq y \leqq 1\}$. For each $\epsilon>0$, let $B_{\epsilon}=X \cap\{(x, y) \mid 0<y<\epsilon\}$. Let a subset $A$ of $X$ be called open if (i) $A$ is open in the usual Euclidean topology of $X$, and (ii) if $(0,0) \in A$, then $B_{\epsilon} \subset A$ for some $\epsilon>0$. This makes $X$ a locally compact non-Hausdorff space. The elements of $\mathcal{H}(X)$ are the one-element sets, and also the two-element sets of the form $\{(0,0),(x, 0)\}$ for

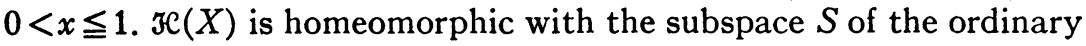
Euclidean plane consisting of the square $X$ together with the closed line segment from $(-1,0)$ to $(0,0)$. The homeomorphism is implemented by the map of $\mathcal{H}(X)$ onto $S$ which sends $\{(x, y)\}$ into $(x, y)$ for $y \neq 0,\{(0,0),(x, 0)\}$ into $(x, 0)$ for $0<x \leqq 1$, and $\{(x, 0)\}$ into $(-x, 0)$ for $0 \leqq x \leqq 1$.

It may be of some interest to give an intrinsic characterization of the Hausdorff compactification. As always, $X$ is a fixed locally compact topological space. Let $K$ be a fixed regular topological space.

Definition. A function $f$ on $X$ to $K$ will be called quasi-continuous if, for each primitive net $\left\{x_{\nu}\right\}$ of elements of $X, \lim _{\nu} f\left(x_{\nu}\right)$ exists in $K$. 
If $X$ is locally compact and Hausdorff, it is easily seen that a function $f$ on $X$ to $K$ is quasi-continuous if and only if (i) $f$ is continuous in the usual sense on $X$, and (ii) $f(x)$ approaches a limit in $K$ as $x$ approaches the point at infinity.

Suppose now that $g$ is a continuous function on $\mathfrak{F C}(X)$ to $K$. For each $x$ in $X$, define

$$
f(x)=g(\langle x\rangle)
$$

By Lemma $2 f$ is quasi-continuous on $X$. Conversely, suppose that $f$ is quasi-continuous on $X$ to $K$. If $Y \in \mathcal{H C}(X)$, by the corollary to Lemma 2 there is a primitive net $\left\{x_{\nu}\right\}$ of elements of $X$ with $Y$ as its limit set. By quasi-continuity, $\lim _{\nu} f\left(x_{\nu}\right)$ exists in $K$; and it is easy to see that $\lim _{\nu} f\left(x_{\nu}\right)$ depends only on $Y$. Let us define $g(Y)=\lim _{\nu} f\left(x_{v}\right)$. Then $g$ is a function on $\mathfrak{H C}(X)$, and (5) holds. In fact, using the regularity of $K$ we see without difficulty that $g$ is continuous on $\mathscr{H C}(X)$. We therefore have:

Theorem 2. Let $X$ be a locally compact topological space, with Hausdorff compactification $\mathfrak{H}(X)$; and let $K$ be any regular topological space. Then (5) sets up a one-to-one correspondence $f \leftrightarrow g$ between the set of all quasi-continuous functions $f$ on $X$ to $K$ and the set of all continuous functions $g$ on $\operatorname{HC}(X)$ to $K$.

Let us specialize $K$ to be (for example) the complex number system. Then the property stated in Theorem 2 uniquely describes $\mathfrak{F}(X)$. More precisely:

Theorem 3. Let $X$ be as in Theorem 2, Z a compact Hausdorff space, and $\Phi$ a mapping of $X$ onto a dense subset of $\mathrm{Z}$ such that the quasicontinuous complex functions on $X$ are precisely the $g \circ \Phi$, where $g$ runs over the continuous complex functions on $\mathrm{Z}$. Then there exists a homeomorphism $F$ of $\mathrm{Z}$ onto $\mathfrak{F}(X)$ such that

$$
F(\Phi(x))=\langle x\rangle \quad(x \in X) .
$$

Proof. By Theorem 2, the space $Q$ of quasi-continuous complex functions on $X$ forms a commutative Banach algebra whose maximal ideal space is homeomorphic to $\mathfrak{F}(X)$. But, according to the hypothesis of Theorem 3 , it is also homeomorphic to $\mathrm{Z}$. Thus $\mathrm{Z} \cong \mathcal{H}(X)$; and $\Phi(x)$ and $\langle x\rangle$ give corresponding maximal ideals.

\section{BibLIOGRAPHY}

1. J. L. Kelley, General topology, Van Nostrand, New York, 1955.

2. Ernest Michael, Topologies on spaces of subsets, Trans. Amer. Math. Soc. 71 (1951), 152-182.

UNIVERSITY OF WASHINGTON 